\title{
Agonist mobility on supported lipid bilayers affects Fas mediated death response
}

\author{
M. Florencia Sánchez ${ }^{\mathrm{a}}$, Valeria Levi ${ }^{\mathrm{b}}$, Thomas Weidemann ${ }^{\mathrm{c}, *}$, Dolores C. Carrer ${ }^{\mathrm{a}, *}$ \\ a Instituto de Investigación Médica Mercedes y Martín Ferreyra (INIMEC), CONICET-Universidad Nacional de Córdoba, Friuli 2434, CC389, 5000 Córdoba, Argentina \\ ${ }^{\mathrm{b}}$ Departamento de Química Biológica-IQUIBICEN, Facultad de Ciencias Exactas, Universidad de Buenos Aires, Ciudad Universitaria, Buenos Aires, Argentina \\ ${ }^{\mathrm{c}}$ Max Planck Institute of Biochemistry, Cellular and Molecular Biophysics, Am Klopferspitz 18, 82152 Martinsried, Germany
}

\section{A R T I C L E I N F O}

\section{Article history:}

Received 23 September 2015

Revised 7 October 2015

Accepted 8 October 2015

Available online $\mathrm{xxxx}$

Edited by A. Chattopadhyay

\section{Keywords:}

Fluorescence correlation spectroscopy

Supported lipid bilayer

Apoptosis

Functionalized surface

Fas receptor

\begin{abstract}
A B S T R A C T
Extrinsic apoptosis is initiated by recognition and clustering of the single-pass transmembrane proteins Fas ligand and Fas expressed at the surface of closely apposed lymphocytes and target cells, respectively. Since Fas-mediated death response was mainly studied with soluble antibodies, the mobility constraints for receptor activation by a membrane embedded agonist is not well understood. We explored this influence by stimulating apoptosis on functionalized supported lipid bilayers, where we quantified agonist mobility by z-scan fluorescence correlation spectroscopy. Using different lipid compositions, we show that the apoptotic response correlates with increased lateral mobility of the agonist in the lipid bilayer.
\end{abstract}

(c) 2015 Federation of European Biochemical Societies. Published by Elsevier B.V. All rights reserved.

\section{Introduction}

Programmed cell death (extrinsic apoptosis) is an active process in which cells disintegrate in an orchestrated and regulated fashion initiated by distinct signalling cascades. In particular Fas receptor (CD95, Apo1) mediated apoptosis is important for tissue maintenance and renewal, embryonic development, the control of cell proliferation in epithelia and the immune system, as well as for pathological situations such as viral infections, autoimmune and neurodegenerative diseases [1-3]. Upon Fas activation, apoptotic cells undergo global morphological changes such as chromatin condensation, loss in cytoskeletal organization, and membrane blebbing [4,5].

Extrinsic apoptosis is initiated by the interaction between the extracellular domains of the single-pass transmembrane receptors Fas and Fas ligand (FasL) differentially expressed at the surface of target cells and natural killer lymphocytes, respectively. The specific cell-cell interaction triggers the formation of a cytotoxic

Author contributions: M.F.S. performed experiments and analyzed data; T.W. and D.C.C. contributed on the conception and design of the study; M.F.S., T.W., V.L. and D.C.C. performed the analysis and interpretation of the data, M.F.S., T.W., V.L. and D.C.C. wrote the manuscript.

* Corresponding authors. Fax: +54 3514695163 (D.C. Carrer).

E-mail addresses: weidemann@biochem.mpg.de (T. Weidemann), dolorescarrer @immf.uncor.edu (D.C. Carrer). synapse characterized by the lateral segregation of membrane receptors and the subsequent concentration of various signalling components at the contact site [6,7].

Fas is thought to preassemble in trimeric form in the target cell membrane [8]. After binding of FasL or agonist antibodies, the complexes rapidly form higher-order aggregates in the membrane and recruit the Fas-associated protein (FADD) via the death domain. Together with pro-caspase 8 and pro-caspase 10 these proteins forms the Death-Inducing Signalling Complex (DISC) [9]. Following DISC formation, oligomerization of the receptor occurs to form larger platforms of receptor aggregates called "caps". Internalization of receptor clusters seems to play a quantitative role for accumulating DISC components which in turn activate effector caspases that lead the cell irreversibly to apoptosis [10].

The biophysical aspects of Fas mediated pathway activation are complex. Different studies have demonstrated that (1) Fas is recruited to lipid raft domains upon activation [11,12], (2) partitioning of FasL into lipid rafts is relevant for apoptosis [13], and (3) the activation of Fas depends crucially on the local lipid composition constituting the cell membrane [14]. For example, it was shown that oligomerization within lipid rafts is facilitated by the formation of ceramide $[15,16]$, a specific lipid with an exceptionally high melting temperature that promotes lateral segregation in membranes [17]. In addition, the partitioning of Fas/FasL oligomers into lipid rafts seems to be a prerequisite for the activation of acid sphingomyelinase, an essential enzyme for producing 
ceramide [16]. Thus, several lipid-related mechanisms constitute positive feedback loops that act in concert for successive DISC formation and a stable activation of the Fas mediated death response. However, due to their interdependence, not all of these processes have been sufficiently characterized.

So far, apoptosis has mainly been studied with soluble inducers such as recombinant extracellular domains or antibodies $[10,18,19]$. However, inducing apoptosis in bulk solution does not fully reproduce the dynamical aspects of the apposed plasma membranes that constitute the physiological cytotoxic synapse. Recently, supported lipid bilayers (SLBs) functionalized with Fas agonists were introduced as a promising model system to study membrane confined Fas activation mechanisms [20]. Using DOPC as a carrier, these authors show that apoptosis triggered at the cell-SLB interface requires a sustained formation of Fas microdomains.

Following this approach, we explore the relevance of membrane fluidity on apoptosis. We functionalized supported lipid bilayers (SLBs) of defined lipid compositions with a Fas antibody fragment that acts as an agonist [21,22]. Using z-scan fluorescence correlation spectroscopy (z-scan FCS) we determine the agonist mobility in different SLBs, and show that the lateral mobility correlates with its efficiency to induce apoptosis in HEK293 and derived cell lines. These observations recapitulate the crucial role of lipid reorganization and membrane fluidity for extrinsic Fas mediated apoptosis.

\section{Materials and methods}

\subsection{Preparation of functionalized SLBS}

Bilayers of dioleoylphosphatidylcholine (DOPC), dipalmitoylpho sphatidylcholine (DPPC) and DOPC:cholesterol (Chol) mixtures (75:25) were prepared by the vesicle fusion method [23]. Briefly, a $1 \mathrm{mM}$ solution of lipids (Avanti Polar Lipids, Alabaster, AL, USA) containing $0.25 \mathrm{~mol} \%$ of biotinylated phosphatidylethanolamine (PE-B), $2 \mathrm{~mol} \%$ of brain phosphatidylserine (BrainPS), and 0.1\% (cellular imaging) or $0.01 \mathrm{~mol} \%$ (z-scan FCS) of the fluorescent lipid analogue 3,3'-dilinoleyloxacarbocyanine perchlorate (DiO-C18) (Life Technologies, Molecular Probes, Eugene, OR, USA) in chloroform: methanol (2:1) as a solvent was evaporated first under nitrogen and then under vacuum, each for $3 \mathrm{~h}$. The formed lipid films were re-hydrated in PBS to a final concentration of $1 \mathrm{mM}$, resuspended by vortexing, and sonicated to obtain small unilamellar vesicles (SUVs). To prepare SLBs, $150 \mu \mathrm{l}$ SUVs supplemented with $3 \mu \mathrm{l} 0.1 \mathrm{M} \mathrm{CaCl}_{2}$ were precipitated for $15 \mathrm{~min}$ on glass in 8 -well Lab-Tek (Nunc, USA) or home-made chambers, and washed four times with $400 \mu \mathrm{l}$ PBS. Hydration, sonication, and liposome incubation steps were performed above the melting temperature of the respective lipid: $25^{\circ} \mathrm{C}$ for DOPC and DOPC:Chol, $50{ }^{\circ} \mathrm{C}$ for DPPC.

To functionalize SLBs, the surfaces were sequentially incubated for $30 \mathrm{~min}$ with labelled streptavidin-AlexaFluor555 (SA*) (Molecular Probes) and Fas agonist ( $\alpha$ Fas, biotinylated (Fab) $)_{2}$ fragment of anti CD95 [DX3], AbCam, Cambridge, MA, USA); both proteins at concentrations ranging from 0.01 to $1 \mu \mathrm{g} / \mathrm{mL}$ depending on well size. After each step samples were washed twice with PBS. For apoptosis quantification by $\mathrm{AnnV}$, we followed the same protocol except that we used non-labelled streptavidin (SA) (Molecular Probes). Surface bound biotinylated $\alpha$ Fas, was detected with $1 \mu \mathrm{g} / \mathrm{mL}$ secondary antibody ((Fab) $)_{2}$ fragment of anti-mouse IgGAlexaFluor546, Molecular Probes).

\subsection{Imaging and FCS}

Measurements were performed on a confocal laser scanning microscope Olympus FV1000 using a 60X NA 1.4 oil-immersion objective. For $488 \mathrm{~nm}$ excitable dyes (GFP, DiO), a 510-540 nm band pass filter was used for detection whereas $543 \mathrm{~nm}$ excitable dyes (AlexaFluor555, AlexaFluor546, Dil) were detected using a 565-610 nm filter. For FCS, intensity traces (18-30 s) were obtained using the pseudo-photon counting mode of the detector. Autocorrelation curves were fitted with a single two-dimensional diffusion model [24].

\subsection{Z-scan FCS}

To avoid positioning issues, we applied z-scan FCS [25-30] an extension of classic FCS in which fluorescence autocorrelation curves are measured at different positions along the optical axis (5-8 steps of $0.3 \mu \mathrm{m}$ ). The diffusion coefficient can be obtained from these data following the procedure described in [24]. Particularly, the residence times $\left(\tau_{\mathrm{D}}\right)$ are related to the distance between the sample plane and the beam waist $w_{0}(\Delta Z)$ as follows:

$$
\tau_{\mathrm{D}}=\frac{w_{0}^{2}}{4 D}\left(1+\left(\frac{\lambda \Delta Z}{\pi w_{0}^{2}}\right)^{2}\right)
$$

where $\lambda$ represents the excitation wavelength. At least fifteen curve sets were evaluated for each type of SLB (Table 1).

\subsection{Cell treatments}

To generate a human cell line expressing FasR-eGFP, we used Flp-recombinase mediated genome insertion into modified HEK293 cells (ATCC, \#CRL-1573) that contained a single landing site (FlpIn system, Life technologies, Invitrogen, Carlsbad, CA, USA). The Fas coding sequence was PCR amplified from phAPO$1 \mathrm{~L}$ (kindly donated by Peter Krammer, DKFZ Heidelberg) and introduced into pcDNA5 ${ }^{\mathrm{TM}} /$ FRT via the unique restriction sites KpnI and ApaI. The insert was assembled by subcloning through intermediate vectors providing the required tags (EGFP, Flag-tag). The resulting open reading frame codes for 595 amino acids (aa \#1-595), comprised of an N-terminal Fas (\#1-335; GI:329299085), a short linker (\#336-339, AAAR, single letter code), the EGFP (\# 340577; GI:1377911), a short linker (\# 578-587; PSTLDSSGSG), followed by a Flag-tag (\# 588-595; DYKDDDDK). Genome insertion in 293 Flp-In cells was performed by transient co-expression of pOG44 coding for the Flp-recombinase and subsequent Hygromycin B (Invitrogen) selection. Single cell lines were isolated by dilution cloning.

The selected FlpIn-FasR-eGFP clone was maintained in DMEM with $10 \%$ fetal bovine serum, supplemented with Hygromycin B $(100 \mu \mathrm{g} / \mathrm{mL})$, penicillin, and streptomycin under standard cell culture conditions $\left(5 \% \mathrm{CO}_{2}, 37^{\circ} \mathrm{C}\right.$ ) (cell culture material from Life Technologies, Invitrogen). For apoptosis induction, $3 \times 10^{4}$ FasReGFP cells per $\mathrm{cm}^{2}$ were plated on top of pre-formed, functionalized SLBs. In experiments involving the soluble agonist, $\alpha$ Fas was added in the absence of SLBs at the time of cell seeding, in chambers pre-incubated $\left(30 \mathrm{~min}\right.$ at $37^{\circ} \mathrm{C}$ ) with $10 \mu \mathrm{g} / \mathrm{mL}$ human fibronectin/PBS (Invitrogen). Cells were allowed to settle for at least $6 \mathrm{~h}$ prior to imaging.

\subsection{Apoptosis detection}

6 or $12 \mathrm{~h}$ post-stimulation, live cells in a 8-well chamber (LabTek) were stained for 15 min with $2 \mu$ l Annexin V-AlexaFluor568 conjugate (AnnV, Molecular Probes) solution in $100 \mu$ proprietary binding buffer, aspirated, and imaged immediately in fresh DMEM. Washing steps were omitted to avoid a loss of apoptotic cells. Images were background-corrected and contrast-enhanced using specific software (ImageJ, NIH). FlpIn cells were counted by visual inspection of fluorescent images. In the case of unlabelled HEK 
cells, the nuclei probe DRAQ5 (Cell Signaling, Danvers, MA, USA) was added to stain the cells. Conditions were tested at least in triplicate, $(n>200)$.

For immuno- and TUNEL-staining the stable FasR-eGFP cells were plated on fibronectin coated coverslips, stimulated by soluble $\alpha$ Fas agonist for $6 \mathrm{~h}$, and fixed with $4 \%(\mathrm{w} / \mathrm{v}$ ) paraformaldehyde in PBS (pH 7.4) for $30 \mathrm{~min}$. at room temperature (RT). Cells were permeabilized with $0.2 \%$ Triton for $5 \mathrm{~min}$. and incubated with $5 \%(\mathrm{v} / \mathrm{v})$ horse serum (Invitrogen) in PBS for $45 \mathrm{~min}$. PARP staining was done sequentially with primary antibody anti-Cleaved PARP (Cell Signaling, Danvers, MA, USA) and an AlexaFluor568-labelled secondary antibody (Invitrogen), each at RT for $1 \mathrm{~h}$. For TUNEL staining (Roche Applied Science, Mannheim, Germany), the reaction mixture was applied to the fixed permeabilized cells for $1 \mathrm{~h}$ at $37^{\circ} \mathrm{C}$ under light protection. Samples were washed with PBS after each step. For cell imaging, cover slips were mounted on glass slides with FluorSave mounting media (Merck, CalBiochem, Darmstadt, Germany).

\section{Results and discussion}

\subsection{Functionalized SLBs for Fas mediated apoptosis}

To mimic the membrane-membrane interface that triggers Fasmediated apoptosis, we developed a model system composed of SLBs and adherent cells. To vary the SLB fluidity, we used either pure unsaturated (DOPC) or a binary mixture of lipids (DOPC:cholesterol) that exhibits a higher viscosity while staying homogeneously mixed at RT. [31]. As an example for drastically impaired diffusion, we further included SLBs of pure saturated phospholipids (DPPC) that are in a quasi-static gel phase at RT in contrast to the fluid SLBs mentioned before [17].

The SLBs were functionalized with a Fas specific antibody agonist ( $\alpha$ Fas) making use of the strong biotin-streptavidin interaction (Fig. 1A). To achieve reproducible agonist surface densities, we administered both streptavidin (SA) and biotinylated agonist under saturating conditions followed by extensive washing. Each of the
A

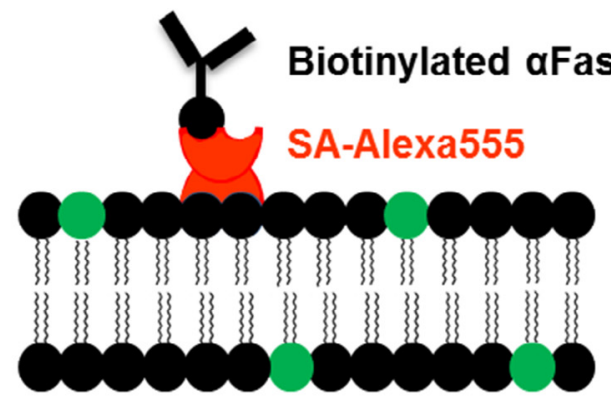

SLB: DOPC, PE-B, DiO-C18

B

DiO-C18

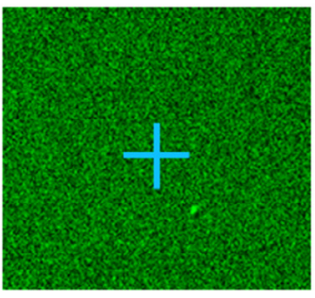

D DiO-C18

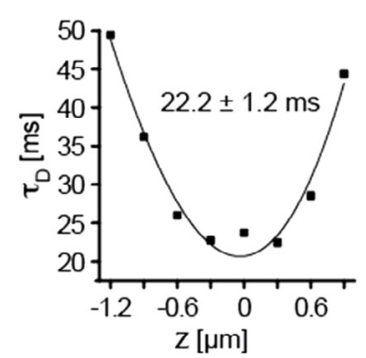

SA-Alexa555

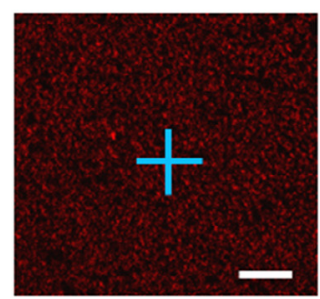

SA-Alexa555

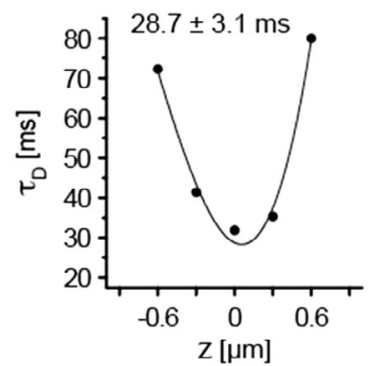

C
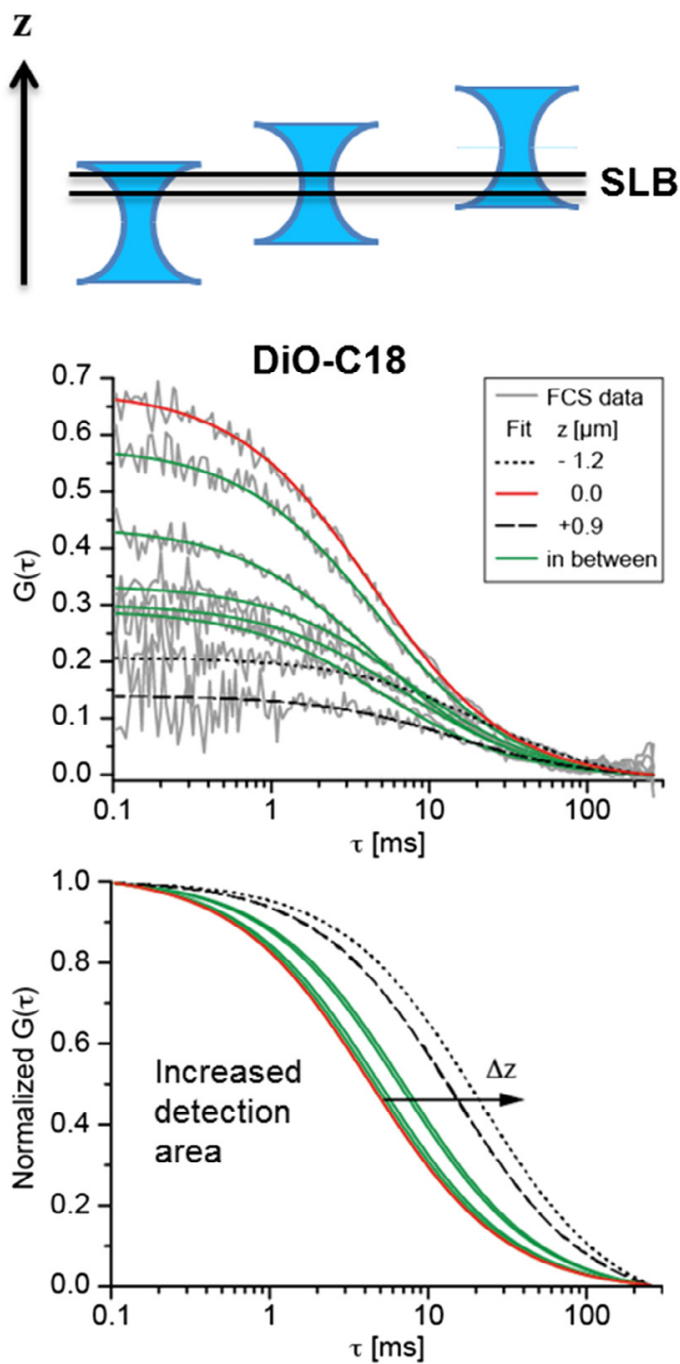

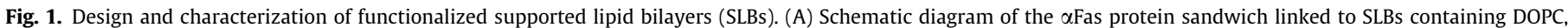

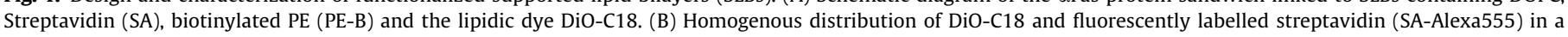

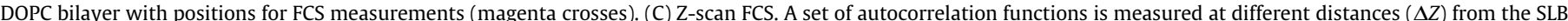

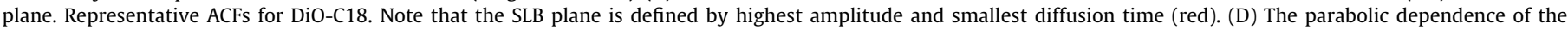
diffusion time $\left(\tau_{\mathrm{D}}\right)$ with $\Delta Z$ allows the determination of the diffusion coefficients. 
binding steps showed a high degree of specificity (Suppl. Fig. S1). As such, the concentration of biotinylated phosphatidylethanolamine (PE-B) in the SLB is limiting and surface densities of SA scaled well with the amount of introduced PE-B in the lipid mixture (Suppl. Fig. S2). We additionally confirmed the homogeneous spatial distribution of SA within SLBs, indicating the absence of protein-mediated large scale aggregations prior to cellular Fas binding (Fig. 1B).

The mobility of the streptavidin/agonist complex within these lipid bilayers was precisely determined by z-scan FCS, a method that overcomes the difficulty of accurately focusing the laser at the SLB plane (Fig. 1C). Z-scan FCS measures the dependence of the mean diffusion times with the axial position of the laser focus (Fig. 1D). These data is then fitted with Eq. (1) to obtain the diffusion coefficient of the protein complex. As a control, we also measured the diffusion coefficient of the membrane probe DiO-C18 in the different bilayers and found that they agreed well with those reported in the literature (Table 1) [32].

In DOPC, the diffusion coefficients of DiO, the complex of PE-B with Alexa555-labelled streptavidin $\left(\mathrm{SA}^{*}\right)$, and the complete protein sandwich containing the agonist $\alpha$ Fas were $\sim 5.0 \mu \mathrm{m}^{2} / \mathrm{s}$ (Table 1); the order of magnitude agreed well with the literature $[26,33]$. As expected, addition of cholesterol decreased the diffusion coefficients by a factor of $4-5[26,34]$. In the presence of cholesterol, binding of the agonist antibody to SA* slightly decreased the mobility, which may indicate weak interactions between the protein and the lipid bilayer surface. In contrast, SLBs composed of DPPC show such a high viscosity that photobleaching

Table 1

Diffusion coefficients of DiO-C18 and the lipid anchored Fas agonist in SLBs of different lipid compositions. Data represent the mean \pm S.E.M.; $n \geqslant 15$.

\begin{tabular}{llll}
\hline $\begin{array}{l}\text { Diffusion coefficient } \\
{\left[\mu \mathrm{m}^{2} / \mathrm{s}\right]}\end{array}$ & DiO-C18 & SA-Alexa555 & SA-Alexa555/ $\alpha$ FasR \\
\hline DOPC & $5.51 \pm 0.18$ & $5.19 \pm 0.19$ & $5.20 \pm 0.28$ \\
DOPC:BrainPS (95:5) & $5.35 \pm 0.28$ & n.d. & n.d. \\
DOPC:Chol (75:25) & $1.34 \pm 0.03$ & $1.40 \pm 0.04$ & $1.06 \pm 0.16$ \\
\hline
\end{tabular}

and other issues related to the very long dwell times the fluorescent molecules spend within the illumination volume prevent the application of FCS [26]. Thus, z-scan FCS showed that the surface bound agonist $\alpha$ Fas exhibits two-dimensional diffusion properties that are dominated by the lipid anchors.

\subsection{Stimulation of Fas-mediated apoptosis}

To generate a microscopic readout for apoptosis, we used a HEK293 derived cell line stably expressing a GFP-tagged Fas construct. This cell line allows monitoring of receptor agreggations at a single cell level of the receptor aggregation induced during apoptosis. Confocal images taken at the bottom plasma membrane show large regions of homogeneously distributed receptors and membrane ruffles, as typical features for adherent cells (Suppl. Fig. S3A). The modified cells expressed most of the fluorescence at the plasma membrane indicating efficient transport of FasReGFP through the constitutive secretory pathway (Suppl. Fig. S3B). Within the cell population, the expression levels of GFP-tagged Fas varied by one order of magnitude (Suppl. Fig. S3C).

Cell adhesion onto SLBs requires either micro-structured fibronectin support [35] or trace amounts of anionic lipids [36]. Therefore we supplemented the SLB mixtures with $2 \%$ of phosphatidylserine (PS), which had negligible effect on the viscosity of the lipid mixture (Table 1). The morphology of the Flp-In FasR-eGFP cells growing on SLBs were indistinguishable from those growing on fibronectin coated glass (Fig. 2A and C).

Stimulation of apoptosis from bulk solution with $\alpha$ Fas produced the expected Fas receptor aggregation in the plasma membrane of cells plated on fibronectin coated coverslips. The morphology of the cells was typical of apoptosis, showing blebbing, receptor internalization and rounded shapes. Patches of GFP-tagged Fas were irregularly distributed all over the cell surface and could therefore be imaged in cross-sections of the cell at any distance from the glass surface (Fig. 2B). In contrast, stimulating cells growing on top of SLBs doped with $\alpha$ Fas, the Fas receptor aggregations were largely confined to the bottom membrane proximal to the support (Fig. 2D). Thus, functionalized SLBs indeed mimic the situation in

\section{Fibronectin coated glass}

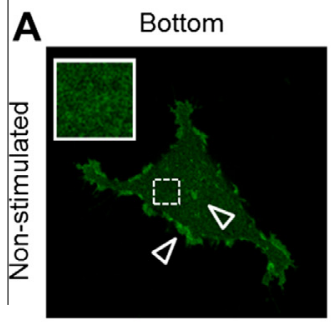

B Bottom

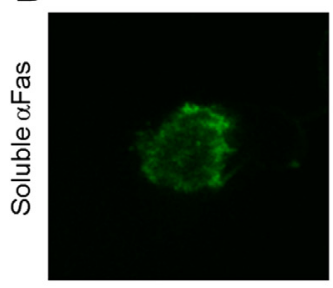

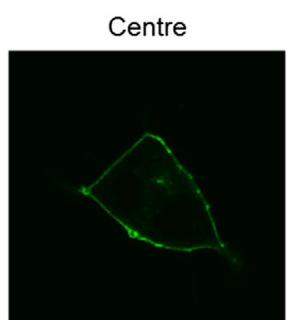

Centre

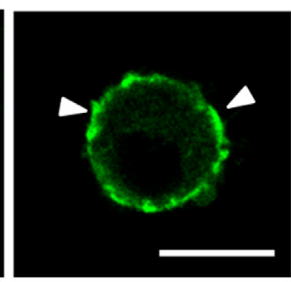

DOPC:BrainPS (98:2) SLB on glass
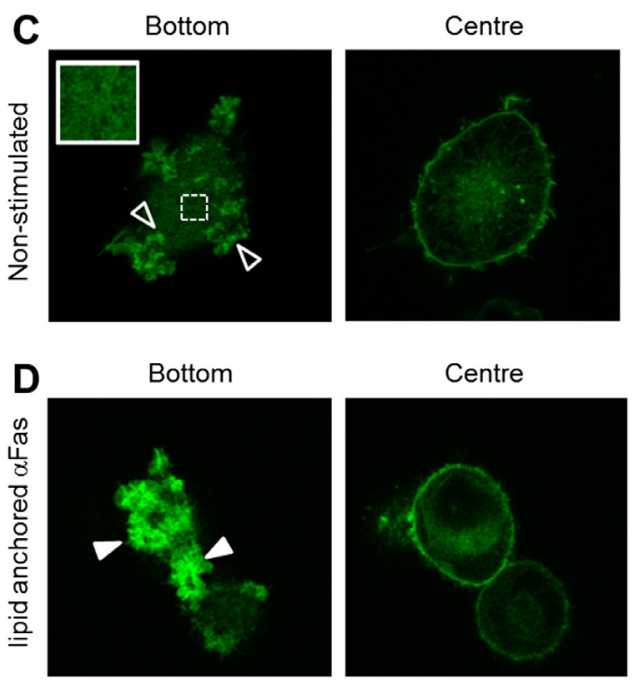

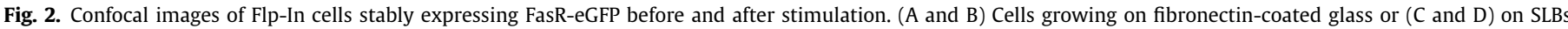

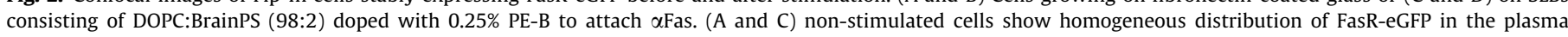

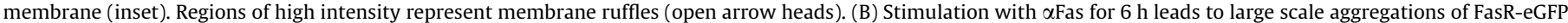

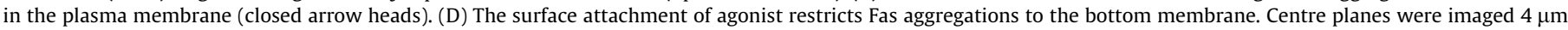
above the bottom plane, scale bar $10 \mu \mathrm{m}$. 


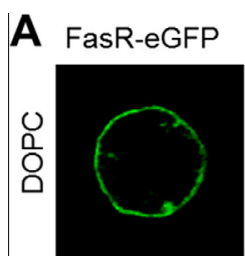

B

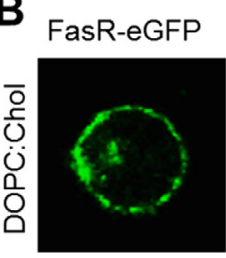

C
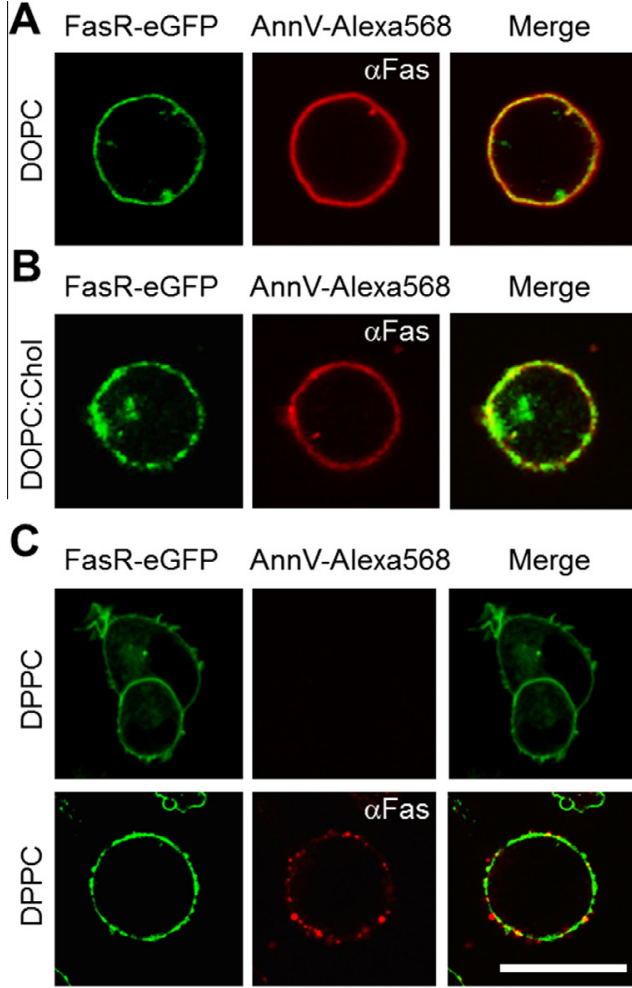

Merge

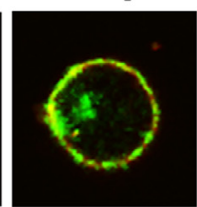

AnnV-Alexa568
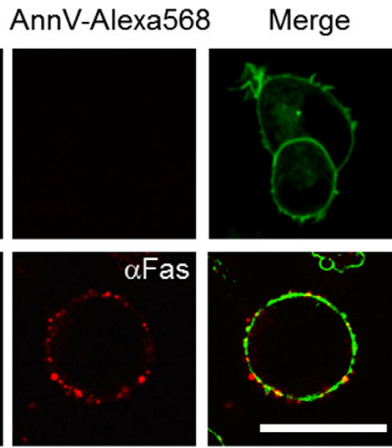

D

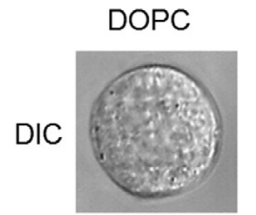

DRAQ5 /

AnnV-Alexa568

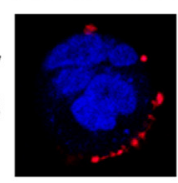

E

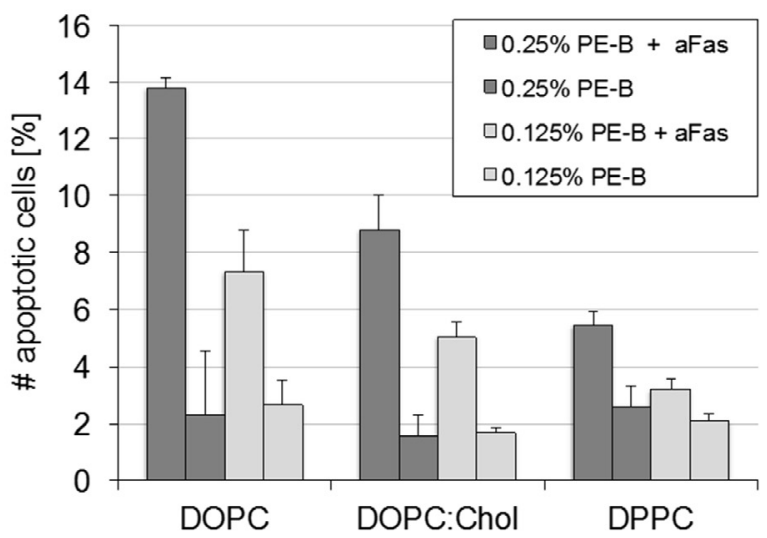

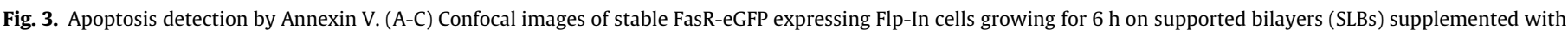

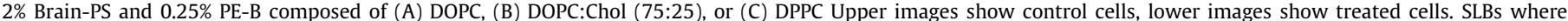

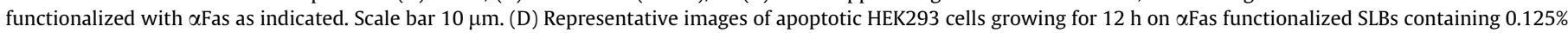

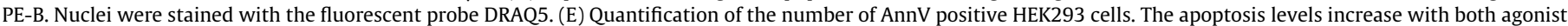
concentrations and fluidity of the SLB.

which apoptosis is induced from tightly apposed membranes. We further confirmed by PARP and TUNEL staining that the death signalling cascade in these cells progressed towards DNA degradation (Suppl. Fig. S4).

\subsection{Apoptosis induced by functionalized SLBs composed of different lipids}

We explored the relevance of membrane fluidity on extrinsic apoptosis by measuring apoptosis induction efficiency in cells plated onto SLBs composed of different lipid mixtures also containing anchored $\alpha$ Fas.

Recent works using soluble agonist showed that the surface density of $\alpha$ Fas should be higher than a threshold (4000 antibodies/cell) for productive signalling in HEK293T cells [19]. Assuming that biotinylated PE binds agonists in a 1:1 relation, the surface density of $\alpha$ Fas would be $\sim 3300$ molecules $/ \mu \mathrm{m}^{2}$ in our experimental conditions. Thus, for a contact area of $10-50 \mu \mathrm{m}^{2}$ we would exceed this threshold by more than an order of magnitude.

Because the SLB surfaces are relatively small $\left(\sim 1 \mathrm{~cm}^{2}\right)$ and prevent the use of detergents for immunostaining, we quantified apoptosis by detecting surface exposed phosphatidylserine (PS) headgroups with fluorescently labelled AnnV [37,38].

Flp-In cells stimulated with the soluble $\alpha$ Fas presented about $24.2 \%$ AnnV positive cells after $6 \mathrm{~h}$, whereas the amount of apoptotic cells growing on functionalized SLBs was significantly reduced (8-15\%). Importantly, the number of apoptotic cells was significantly higher in the assayed condition with respect to the corresponding non-treated controls ( $\sim 5 \%)$, suggesting that $\alpha \mathrm{Fas}$ protein induces cell death in a significant fraction of cells (Suppl. Fig. S5). Visual discrimination of AnnV positive cells from nonstained cells was obvious, despite the diverse cell morphologies associated with the progression of apoptosis: rounded homogeneously stained plasma membranes (Fig. $3 \mathrm{~A}$ and B), blebs, or patches. Interestingly, none of the cells growing on gel-like DPPC bilayers showed a homogeneous staining; the AnnV signal was concentrated in speckles at the cell periphery that seem to be excluded from FasR-eGFP aggregates (Fig. 3C). These data also suggest that the agonist mobility plays a role in apoptosis induction since this process was more efficient in DOPC bilayers in comparison to DOPC:Chol or DPPC.

To test this hypothesis under more physiological Fas expression levels we seeded HEK293 cells $[1,19]$ onto the different SLBs for $12 \mathrm{~h}$ and stained with AnnV as described before. Fig. 3D shows that the staining produced patchy signals associated with a distinct subpopulation of cells for all three types of SLBs. Quantification yielded slightly reduced apoptosis levels of about $6-14 \%$ above a background level of only $2 \%$ (Fig. 3E), presumably reflecting the much lower Fas expression levels. However, in agreement with the Flp-In cells, the apoptosis response in native HEK293 cells clearly correlated with the fluidity of the SLB and hence the lateral, two-dimensional mobility of $\alpha$ Fas (Table 1 ) in a concentration dependent manner.

\section{Conclusions}

To mimic cell-cell mediated extrinsic apoptosis, we have generated supported lipid bilayers of different lipid composition that were functionalized with a surface attached Fas-specific antibody. Applying z-scan FCS we showed that the biotinylated lipid anchor conveys the dynamic properties of the membrane to the streptavidin-linked agonist. For example, reducing membrane fluidity by addition of cholesterol reduced the diffusion coefficients of 
lipid and protein to the same degree. Thus, streptavidin or agonist binding has, if at all, only a small effect on mobility, which can be explained by the negligible hydrodynamic drag introduced by the protruding protein domains in the aqueous phase [26,39].

We showed that a significant fraction of membrane-anchored agonist remains biologically functional since cells expressing GFP-tagged Fas construct showed the expected DISC formation and large-scale aggregations that correlated with an increased response towards classic apoptosis markers (PARP, TUNEL, AnnV). Moreover, quantification of the fraction of apoptotic cells revealed a striking dependence on the fluidity of the membrane support for both Fas overexpressing Flp-In cells and HEK293 cells.

Our observations are in line with the canonical model that proposes aggregation of trimeric Fas/FasL complexes as a stringent prerequisite for extrinsic apoptosis. Using Jurkat cells interacting with functionalized SLBs, Zhang et al. recently showed that Fas/ FasL microdomains form as early as 10 min after seeding the cells [20]. These microdomains were stably maintained for at least an hour, the time at which AnnV signals started to emerge. Shifting the approach to later time points, we can confirm a strong correlation between large scale Fas aggregations in the plasma membrane and AnnV signals in Flp-In and HEK293 cells. While it may be expected that such aggregation phenomena depend on translocation of the membrane spanning Fas/FasL complexes, and hence diffusion within the plasma membrane, we are not sure at this point why decisions towards apoptosis should be kinetically controlled also for longer stimulation times $(6-12 \mathrm{~h})$. One possible scenario is that an increased lateral diffusion could act as a mechanism to concentrate SLB-linked agonists at the cell-bilayer-contact site. Thus, Fas molecules would be continually engaged at times after the initial contact of the cells with the membrane.

Our knowledge about the time evolution of Fas-mediated signalling is still rather incomplete. The superposition of molecular processes involves many factors and spans time scales ranging from seconds to hours [9]. For example, Fas association of cytoplasmic DISC components, e.g. caspase- 8 fragments, can be detected as early as one second after Fas crosslinking [40]. A cellular decision between extrinsic apoptosis or, in contrary, activation of the growth promoting NFKB pathway in HeLa cells seems to takes place in the first hour post-stimulation [41]. Therefore it is likely that the quantified apoptosis response observed in our work routes back to a sensitive initial phase after seeding. Since we kept the cells under continuous stimulation, it is surprising that membrane fluidity has such dramatic effects in the number of apoptotic cells; for cells that are stimulated for long periods of time (6-12 $\mathrm{h}$ ) there seems to be sufficient time to potentially assemble a functional DISC. Thus, our data point towards the existence of more complex long-term regulatory cues.

Taken together, we show here that membrane fluidity plays an important role for the regulation of Fas-mediated extrinsic apoptosis, an intriguing mechanism that has been recently proposed also for other important immunological signalling pathways [42,43].

\section{Acknowledgements}

M.F.S. was a PhD Fellow of SeCyT-UNC belonging to the PRH program of ANPCyT-UNC while performing this work. V.L. and D. C.C. are Career Researchers from CONICET. This work was financially supported by SeCyT-UNC, ANPCyT Young Researchers Grant (PICT-2008-0171), ANPCyT-PRH Grant (PICT 76) and Alexander von Humboldt Foundation Alumni Equipment Grant to D.C.C., ANPCyT (PICT 2008-1104) and UBACyT (20020110100074) grants to V.L. and PFDT grant to MFS. We thank Prof. Peter H. Krammer for gently providing the Apo-1 plasmid. We also thank Prof. Manuel Prieto for valuable discussions and Prof. Enrico Gratton for his generosity in teaching us how to perform FCS measurements with the Olympus FV1000 microscope. The software used to analyze FCS data was generously provided by Dr. Jonas Ries.

\section{Appendix A. Supplementary data}

Supplementary data associated with this article can be found, in the online version, at http://dx.doi.org/10.1016/j.febslet.2015.10. 009.

\section{References}

[1] Li-Weber, M. and Krammer, P.H. (2003) Function and regulation of the CD95 (APO-1/Fas) ligand in the immune system. Semin. Immunol. 15, 145-157.

[2] Falschlehner, C., Schaefer, U. and Walczak, H. (2009) Following TRAIL's path in the immune system. Immunology 127, 145-154.

[3] Depraetere, V. and Golstein, P. (1997) Fas and other cell death signaling pathways. Semin. Immunol. 9, 93-107.

[4] Nagata, S. (1997) Apoptosis by death factor. Cell 88, 355-365.

[5] Feig, C. and Peter, M.E. (2007) How apoptosis got the immune system in shape Eur. J. Immunol. 37 (Suppl. 1), S61-S70.

[6] Smyth, M.J., Cretney, E., Kelly, J.M., Westwood, J.A., Street, S.E., Yagita, H. Takeda, K., van Dommelen, S.L., Degli-Esposti, M.A. and Hayakawa, Y. (2005) Activation of NK cell cytotoxicity. Mol. Immunol. 42, 501-510.

[7] Dustin, M.L. and Long, E.O. (2010) Cytotoxic immunological synapses. Immunol. Rev. 235, 24-34.

[8] Golstein, P. (2000) Signal transduction. FasL binds preassembled Fas. Science 288, 2328-2329.

[9] Peter, M.E. and Krammer, P.H. (2003) The CD95(APO-1/Fas) DISC and beyond. Cell Death Differ. 10, 26-35.

[10] Lee, K.H., Feig, C., Tchikov, V., Schickel, R., Hallas, C., Schutze, S., Peter, M.E. and Chan, A.C. (2006) The role of receptor internalization in CD95 signaling. EMBO J. 25, 1009-1023.

[11] Mollinedo, F. and Gajate, C. (2006) Fas/CD95 death receptor and lipid rafts: new targets for apoptosis-directed cancer therapy. Drug Resist. Updates 9, 51 73.

[12] Hueber, A.O., Bernard, A.M., Herincs, Z., Couzinet, A. and He, H.T. (2002) An essential role for membrane rafts in the initiation of Fas/CD95-triggered cell death in mouse thymocytes. EMBO Rep. 3, 190-196.

[13] Cahuzac, N., Baum, W., Kirkin, V., Conchonaud, F., Wawrezinieck, L., Marguet D., Janssen, O., Zornig, M. and Hueber, A.O. (2006) Fas ligand is localized to membrane rafts, where it displays increased cell death-inducing activity. Blood 107, 2384-2391.

[14] Gajate, C. and Mollinedo, F. (2005) Cytoskeleton-mediated death receptor and ligand concentration in lipid rafts forms apoptosis-promoting clusters in cancer chemotherapy. J. Biol. Chem. 280, 11641-11647.

[15] Cremesti, A., Paris, F., Grassme, H., Holler, N., Tschopp, J., Fuks, Z., Gulbins, E. and Kolesnick, R. (2001) Ceramide enables fas to cap and kill. J. Biol. Chem. 276, 23954-23961.

[16] Grassme, H., Cremesti, A., Kolesnick, R. and Gulbins, E. (2003) Ceramidemediated clustering is required for CD95-DISC formation. Oncogene 22, 5457 5470 .

[17] Carrer, D.C. and Maggio, B. (1999) Phase behavior and molecular interactions in mixtures of ceramide with dipalmitoylphosphatidylcholine. J. Lipid Res. 40, 1978-1989.

[18] Lavrik, I.N., Golks, A., Riess, D., Bentele, M., Eils, R. and Krammer, P.H. (2007) Analysis of CD95 threshold signaling: triggering of CD95 (FAS/APO-1) at low concentrations primarily results in survival signaling. J. Biol. Chem. 282, 13664-13671.

[19] Ranjan, K., Surolia, A. and Pathak, C. (2012) Apoptotic potential of Fasassociated death domain on regulation of cell death regulatory protein cFLIP and death receptor mediated apoptosis in HEK 293T cells. J. Cell Commun. Signal. 6, 155-168.

[20] Zhang, L., Kaizuka, Y. and Hanagata, N. (2012) Imaging of Fas-FasL membrane microdomains during apoptosis in a reconstituted cell-cell junction. Biochem. Biophys. Res. Commun. 422, 298-304.

[21] Carrer, D.C., Kummer, E., Chwastek, G., Chiantia, S. and Schwille, P. (2009) Asymmetry determines the effects of natural ceramides on model membranes. Soft Matter 5, 3279-3286.

[22] Richter, R.P., Berat, R. and Brisson, A.R. (2006) Formation of solid-supported lipid bilayers: an integrated view. Langmuir 22, 3497-3505.

[23] Sui, S.F., Urumow, T. and Sackmann, E. (1988) Interaction of insulin receptors with lipid bilayers and specific and nonspecific binding of insulin to supported membranes. Biochemistry 27, 7463-7469.

[24] Machan, R. and Hof, M. (2010) Lipid diffusion in planar membranes investigated by fluorescence correlation spectroscopy. Biochim. Biophys. Acta 1798, 1377-1391.

[25] Garcia-Saez, A.J., Carrer, D.C. and Schwille, P. (2010) Fluorescence correlation spectroscopy for the study of membrane dynamics and organization in giant unilamellar vesicles. Methods Mol. Biol. 606, 493-508.

[26] Benda, A., Benes, M., Marecek, V., Lhotsky, A., Hermens, W.T. and Hof, M. (2003) How to determine diffusion coefficients in planar phospholipid systems by confocal fluorescence correlation spectroscopy. Langmuir 19 $4120-4126$ 
[27] Ganguly, S. and Chattopadhyay, A. (2010) Cholesterol depletion mimics the effect of cytoskeletal destabilization on membrane dynamics of the serotonin1A receptor: a zFCS study. Biophys. J. 99, 1397-1407.

[28] Humpolickova, J., Gielen, E., Benda, A., Fagulova, V., Vercammen, J., Vandeven, M. Hof, M., Ameloot, M. and Engelborghs, Y. (2006) Probing diffusion laws within cellular membranes by Z-scan fluorescence correlation spectroscopy. Biophys. J. 91, L23-L25.

[29] Miszta, A., Machan, R., Benda, A., Ouellette, A.J., Hermens, W.T. and Hof, M. (2008) Combination of ellipsometry, laser scanning microscopy and Z-scan fluorescence correlation spectroscopy elucidating interaction of cryptdin-4 with supported phospholipid bilayers. J. Pept. Sci. 14, 503509.

[30] Stefl, M., Kulakowska, A. and Hof, M. (2009) Simultaneous characterization of lateral lipid and prothrombin diffusion coefficients by z-scan fluorescence correlation spectroscopy. Biophys. J. 97, L01-L03.

[31] Kahya, N., Scherfeld, D., Bacia, K. and Schwille, P. (2004) Lipid domain formation and dynamics in giant unilamellar vesicles explored by fluorescence correlation spectroscopy. J. Struct. Biol. 147, 77-89.

[32] Bacia, K. Scherfeld, D. Kahya, N. and Schwille, P. (2004) Fluorescence correlation spectroscopy relates rafts in model and native membranes. Biophys. J . 87, 1034-1043.

[33] Przybylo, M., Sykora, J., Humpolickova, J., Benda, A., Zan, A. and Hof, M. (2006) Lipid diffusion in giant unilamellar vesicles is more than 2 times faster than in supported phospholipid bilayers under identical conditions. Langmuir 22, 9096-9099.

[34] Kahya, N and Schwille, P. (2006) How phospholipid-cholesterol interaction modulate lipid lateral diffusion, as revealed by fluorescence correlation spectroscopy. J. Fluoresc. 16, 671-678.
[35] Kam, L. and Boxer, S.G. (2001) Cell adhesion to protein-micropatternedsupported lipid bilayer membranes. J. Biomed. Mater. Res. 55, 487-495.

[36] Groves, J.T., Mahal, L.K. and Bertozzi, C.R. (2001) Control of cell adhesion and growth with micropatterned supported lipid membranes. Langmuir 17, 51295133.

[37] Hu, T., Shi, J., Jiao, X., Zhou, J. and Yin, X. (2008) Measurement of annexin V uptake and lactadherin labeling for the quantification of apoptosis in adherent Tca8113 and ACC-2 cells. Braz. J. Med. Biol. Res. 41, 750-757.

[38] Koopman, G., Reutelingsperger, C.P., Kuijten, G.A., Keehnen, R.M., Pals, S.T. and van Oers, M.H. (1994) Annexin V for flow cytometric detection of phosphatidylserine expression on B cells undergoing apoptosis. Blood 84, $1415-1420$.

[39] Kahya, N., Scherfeld, D., Bacia, K., Poolman, B. and Schwille, P. (2003) Probing lipid mobility of raft-exhibiting model membranes by fluorescence correlation spectroscopy. J. Biol. Chem. 278, 28109-28115.

[40] Kischkel, F.C., Hellbardt, S., Behrmann, I., Germer, M., Pawlita, M., Krammer, P. H. and Peter, M.E. (1995) Cytotoxicity-dependent APO-1 (Fas/CD95)associated proteins form a death-inducing signaling complex (DISC) with the receptor. EMBO J. 14, 5579-5588.

[41] Neumann, L., Pforr, C., Beaudouin, J., Pappa, A., Fricker, N., Krammer, P.H., Lavrik, I.N. and Eils, R. (2010) Dynamics within the CD95 death-inducing signaling complex decide life and death of cells. Mol. Syst. Biol. 6, 352.

[42] Hsu, C.J., Hsieh, W.T., Waldman, A., Clarke, F., Huseby, E.S., Burkhardt, J.K. and Baumgart, T. (2012) Ligand mobility modulates immunological synapse formation and T cell activation. PLoS One 7, e32398.

[43] Ketchum, C, Miller, H., Song W and Upadhyaya, A. (2014) Ligand mobility regulates B cell receptor clustering and signaling activation. Biophys J. 106, $26-36$. 